\title{
WFORM: A graphical speech-waveform editing and analysis system
}

\author{
GEORGE J. BOGGS and MICHAEL D. CONNELLY \\ GTE Laboratories, Waltham, Massachusetts
}

\begin{abstract}
A graphical waveform editor for use in speech research applications is described. The editor operates in 32 kwords of main memory on a DEC LSI-11/23 microcomputer under the RSX-11M operating system. The graphics subsystem is a Hewlett-Packard vector graphics controller and display unit. The editor is written in ANSI standard FORTRAN IV to promote portability. WFORM is capable of reading speech files and displaying the contents of these files in either visual or auditory format. In addition, the editor provides the capability to excise arbitrary portions of speech files, concatenate several speech files, and modify the contents of speech files by windowing. Speech analysis is also available in the form of the fast Fourier transform, and a number of windowing techniques are useful for Fourier analysis. The results of the Fourier transform can be displayed either in the form of power spectra or as a rough spectrograph.
\end{abstract}

Recent interest in research requiring digital speech recording and/or synthesis has generated a need for general-purpose software that provides digital speechediting facilities. In particular, a speech-waveform editor that operates on integer data of the form described by Boggs, Alley, and Guzdial (1984) or Klatt (1979) is useful for such research. In this paper, we describe the operation and structure of a speech-waveform editor designed for research purposes.

The software described here is written in ANSI standard FORTRAN IV to promote portability, and it runs on a Digital Equipment Corporation (DEC) LSI-11/23 microprocessor running the RSX 11-M (Version 4.0) operating system. The software, as written, requires the use of the Hewlett-Packard (H-P) 1351A vector graphics controller, the H-P 1311B vector graphics display unit, a DEC DRV-11J parallel I/O interface board, and a 12bit bipolar two's-complement digital-to-analog convertor (DAC).

Several functional and useful waveform editors already exist. System dependence in terms of graphics equipment, data transfer, and DAC facilities is an apparently unavoidable characteristic of the editors that are low in cost. This editor is obviously no exception. However, the graphics, data communications, and DAC facilities operate on a fairly simple set of rules and instructions that are replicated or imitated to a greater or lesser degree by other generally available and affordable hardware, and system portability is generally quite straightforward. In addition, the minimal memory requirements of this editor (32 kwords) makes it practical to perform waveform editing on small systems for which such a possibility did not previously exist. Hence, the software can be regarded as

The authors' mailing address is: Human Factors Department, Telecommunications Research Laboratory, GTE Laboratories, Inc., 40 Sylvan Road, Waltharn, MA 02254. a practical approximation to the general problem of editing speech waveforms in the psychological laboratory with a minimum of investment. One unfortunate, but unavoidable (given the constraints of RSX-11M), aspect of the WFORM is that the editor only accommodates $1 \mathrm{sec}$ of speech. For less restrictive operating systems, this limitation can be overcome simply by increasing the array size of a single variable and by slightly altering the $\mathrm{x}$ axis labeling and file-read routines.

Finally, it was a major goal in the software development effort to produce an editor that is easy to use and maintain and to incorporate a variety of options useful for psychologically oriented speech research. The user interface is menu driven, and (nearly) mistake proof. All options available to the user at any time are fully documented on-line, and typing errors (other than those that cause the operating system to fail) are correctable and nonfatal. For maintainability, the program is internally documented and modular. This makes it straightforward for users with FORTRAN programming experience to understand and modify the code if necessary.

Although the software interface has been designed for the naive user, the software does not pretend to be a tutorial on speech-waveform analysis. Some knowledge of speech and signal analysis (e.g., the utility of the discrete Fourier transform) is presumed and is necessary to obtain maximum benefit from the system.

The operation of the waveform editor is depicted in the block diagram shown in Figure 1. Global user commands are processed through the main module, WFORM, and appropriate modular subroutines are called from WFORM to perform specific functions. There are a number of these subroutines, described later in the paper, and they are accessed via the overlay capability of RSX-11M. The speech waveform resides in a common block denoted /WAVE/. The various modular subroutines called by WFORM perform graphical, analytic, and playback functions on the 


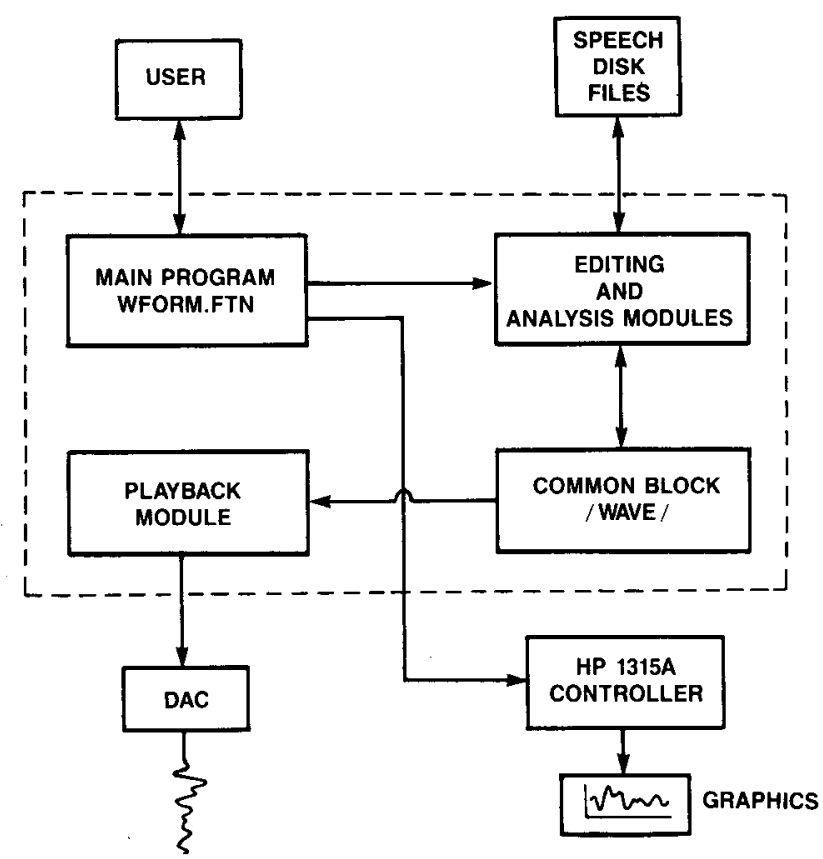

Figure 1. Block diagram of the software structure for WFORM.

data contained in /WAVE/, but cannot manipulate the data directly. The common block /WAVE/ contains a maximum of $1,000 \mathrm{msec}$ of continuous speech (assuming a sampling rate of $10 \mathrm{kHz}$ ).

The graphical instructions that control the plotting of vectors on the graphics-display subsystem are not sent directly to the display from the FORTRAN modules. The graphics instructions are sent to the H-P 1351A controller (via the DRV-11J parallel interface). After receiving the graphics instructions, the H-P 1351A modifies and maintains the graphics display. This feature is particularly useful on small and/or multiuser computer systems, because the computing resources necessary to maintain the display are not drawn from central processing resources.

\section{GENERAL DESCRIPTION}

The various utilities found in the editor can be roughly divided into two categories. The first category includes utilities that perform actual editing on waveform files. These utilities display the waveform (or specified portions of the waveform) on the graphics display, save portions of the waveform in temporary (or scratch) files, and create new permanent files containing waveforms composed of waveforms contained in temporary files. The second category includes utilities that perform various analytical and mathematical operations on waveforms. These include waveform scaling for display (e.g., volts or decibels), discrete Fourier transforms, and various windowing functions.

At no time in any of the utilities is the basic waveform that is stored in /WAVE/ physically changed in any way. All results are provided either visually, aurally, or on disk storage. Any sequence of functions can therefore be per- formed without alteration to the original waveform data. This feature precludes having to reenter the same waveform file after an unsuccessful editing attempt. It is possible, however, to enter a new permanent waveform file after returning to the main menu or after creation of an edited temporary file.

\section{FILE EDITING UTILITIES}

Psychologically oriented speech research often requires a software facility that permits the creation of new speech files that are nothing more than concatenated segments of other files. This waveform editor allows flexibility in concatenating waveform files. The editor assumes that all waveform data is in the range of 4,095 to 0 , corresponding to DAC voltages of $-5 \mathrm{~V}$ to $+5 \mathrm{~V}$, respectively.

When WFORM is invoked, the user specifies an input file containing the waveform to be edited. WFORM then reads the contents of the waveform file into memory and displays the entire waveform in a plot of amplitude versus time, as shown in Figure 2. (Figure 2 is a $370-\mathrm{msec}$ display of the vowel /a/ synthesized by the Klatt, 1979, synthesis software.) After display of the waveform file, the user selects one of several options from the main menu. The following sections describe the menu options that perform the editing, splicing, and creation of new waveform files.

\section{Select New Current Waveform}

Whenever a new waveform is read into memory, the visual representation (as shown in Figure 2) appears on the graphics display. The vector plot has 1,024 pixels on both the $\mathrm{x}$ and $\mathrm{y}$ axes. Only 1,000 of the pixels are used for plotting on the $x$-axis to allow room for axis labels. Whenever a waveform is displayed, the $\mathrm{x}$-axis is scaled such that the full extent of the $\mathrm{x}$-axis is used to represent

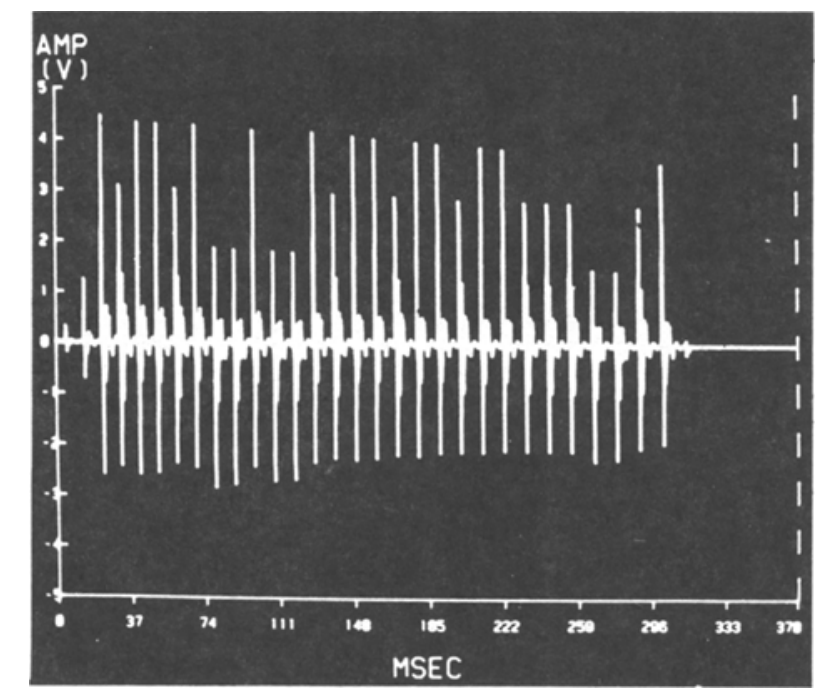

Figure 2. Initial screen display after entering WFORM. The display represents a $370-\mathrm{msec}$ vowel $/ \mathbf{a} /$. 
the duration of the waveform. Because the duration of the displayed waveform is often greater than $100 \mathrm{msec}$ (corresponding to exactly $1 \mathrm{pixel} / \mathrm{sample}$ ), an integer variable (SR) contains the number of samples between successive points. SR is greater than 1 only when the utterance is longer than 1,000 samples or $100 \mathrm{msec}$. This permits a speech sample whose duration exceeds the resolution of the graphics display to be displayed (at the expense of a completely true view of the file).

\section{Display of Selected Endpoints}

The user selects a temporal segment of the current waveform (the contents of /WAVE/) by selecting the appropriate option from the main menu. The user is prompted for the endpoints of the segment to be displayed (in milliseconds). These endpoints specify the temporal window through which the current waveform will be viewed.

The reader should note that temporal endpoints are selected by entering time values rather than by using a movable cursor. A movable cursor is often desirable in waveform editing. In fact, movable cursors were evaluated during the development of this editor; however, this particular configuration of processor, operating system, and graphics peripherals did not permit movable cursor action with satisfying results (in terms of constant cursor velocity).

After the endpoints are specified, a vertical line is plotted on the display at the specified temporal endpoints (on the $\mathrm{x}$-axis). Above each line is displayed the scale units corresponding to the y-axis. An example is shown in Figure 3, where the user specified endpoints of 100 and $200 \mathrm{msec}$ for the waveform plotted in Figure 2. Because the specified endpoints represent points in the file where

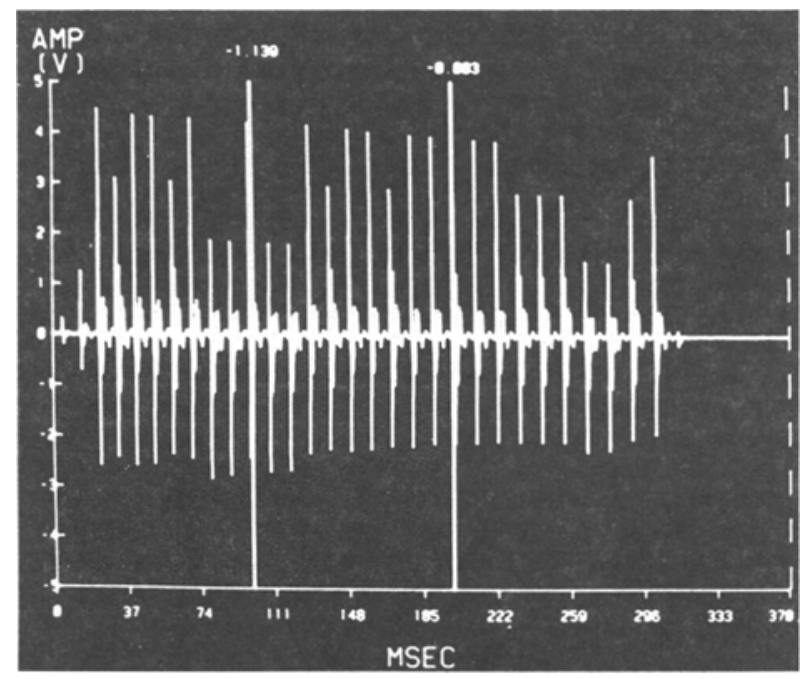

Figure 3. The 370-msec vowel /a/ after the user selected two endpoints for splicing at $100 \mathrm{msec}$ and $200 \mathrm{msec}$. Note the amplitude values displayed above each vertical line segment denoting the endpoints.

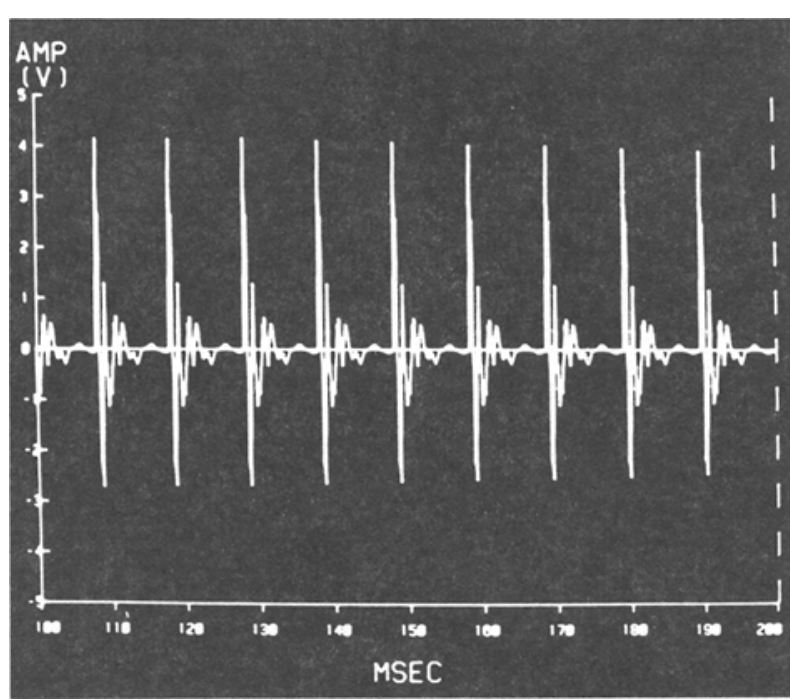

Figure 4. The vowel /a/ expanded within the range $100 \mathrm{msec}$ to 200 msec.

the waveform segment will be delimited, and the density of the samples is only 1 sample per $0.1 \mathrm{msec}$, any part of a user input beyond the first decimal place is simply truncated by the editor. The new display will simply be a temporal segment of the entire waveform, with the extent of the segment being the endpoints specified by the user. The $\mathrm{x}$-axis is also relabeled from the lower to the higher endpoint in 10 increments of $1 / 10$ of the new waveform segment's range.

If either of the temporal boundaries input by the user is less than 0 msec or greater than the total duration of the speech waveform, a message appears on the user terminal requesting a new boundary. If the upper boundary is less than the lower, both boundaries must be reentered. Therefore, the editor will not fail with boundary input errors, and any boundaries that pass through the errorchecking routine must be nominally and ordinally valid.

\section{Display New Current Waveform}

After the user is permitted to specify new waveform endpoints, the new waveform is displayed across the extent of the $x$-axis. Figure 4 shows the new display after the segment selected in Figure 3 is displayed. At this point, the user has the option to return to the main menu. New endpoints may then be specified if the current display is not satisfactory.

\section{Waveform Endpoint Manipulation}

When editing speech, it is often desirable to ensure that the ends of a waveform segment are at zero-volt crossings, because abrupt changes in voltage can cause audible distortion during playback. There are two ways to avoid this problem while editing. First, the editor contains a zero-crossing switch. When this switch is enabled, the first positive-going zero crossing outside the endpoints 
selected by the user is selected as the true endpoint. The state of the zero-crossing switch is set by the user in the main menu (the default state is OFF).

Whether the zero-crossing switch is ON or OFF, the user may specify that a triangular window be imposed on the displayed waveform endpoints. The extent of the window, input by the user in milliseconds, can be in the range $0.1 \mathrm{msec}$ (effectively no window, or a rectangular window) to one half the total duration of the displayed waveform. However, because only one value is entered, the window must be symmetric. The duration of the window is stored in SLOPE, and windowing is accomplished simply by multiplying the first and last SLOPE milliseconds of the waveform by a right triangular function that ranges from zero at the endpoints to 1.0 at SLOPE milliseconds into the displayed waveform. The effects of this operation are shown in Figure 5, with a 20-msec window. Note that multiplications are not performed on the contents of /WAVE/, so the user may return to the original, unchanged waveform. As long as the displayed waveform remains current, however, the windowed version of the waveform is used in further displays, for writing to temporary files or for performing signal analysis.

\section{Create Temporary or Permanent Files}

The counterpart to the routine that specifies a new current waveform is a set of routines that store edited waveforms in either temporary or permanent files. When the user seiects the option to store several waveforms in temporary files, separate files are not actually created for each waveform segment. The waveform segments are written into separate areas of a single temporary scratch file. A direct-access scratch file can be accessed in much the same way as a memory-resident array, and this arrangement is economical in terms of both memory resources and disk resources. After a scratch file has been created, the user

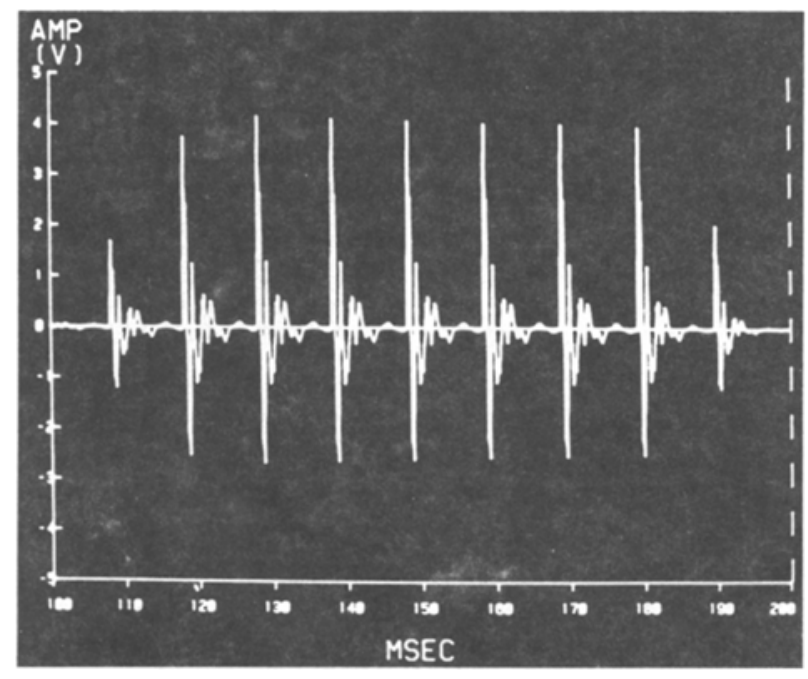

Figure 5. The 100- to 200-msec segment of the vowel /a/ with a 20-msec triangular window. may return to the file he/she was editing with the current waveform unchanged. If this is done, the situation remains exactly as it was before the scratch file was created. Another alternative available to the user is to read an entirely new speech file into the array /WAVE/. If the user chooses to read a new file, the new file becomes the current waveform and overwrites any speech data present in /WAVE/.

The user also has the option to create a permanent file after the scratch file has been created. This option invokes a file-builder routine that creates, opens, and closes a new permanent file. The contents of the new permanent file are a user-specified concatenation of speech segments from the scratch file. Upon selecting the permanent file option, the user is given a list of previously created scratch file segments (note that it is impossible for this list to be empty, since the permanent file option does not become available until at least one speech segment has been written to a scratch file). The user then specifies the order of speech segments to be written to the permanent file.

All menu options in the permanent-file-builder routine cause the permanent file(s) to be closed upon exit from the routine. However, the scratch file, once created, exists only for the remainder of the editing session. The options available to the user upon exit from the permanent file builder are: (1) return to the main menu; (2) read the permanent file just created into /WAVE/, display the file, and exit the editor; and (3) read the new permanent file as in (2) and return to the main menu.

\section{WAVEFORM ANALYSIS UTILITIES}

In addition to the editing features described above, a set of utilities for waveform analysis is available. The analysis routines operate on the waveform being displayed, and not on the contents of /WAVE/. Therefore, any analysis action taken does not alter the data currently residing in /WAVE/.

\section{Select Y-Axis Units}

The user can display the current waveform in units of either volts or decibels on the $y$-axis. If a window has been imposed, the window is accounted for when the $y$ axis units are transformed.

Display in volts involves converting the speech data (which, in our case, are 12-bit integers in the range $0-4,095)$ to voltages in the range $+5 \mathrm{~V}$ to $-5 \mathrm{~V}$. Since our DAC is linear, the voltages are mapped onto the integers using a simple linear relationship (e.g., $+5 \mathrm{~V} .=0$, $-5 \mathrm{~V}=4,095)$.

If a display in decibels is desired, the y-axis units become decibels. See Figure 6 for a display of Figure 2 in decibel units. To perform this conversion, the equation

$$
\mathrm{dB}=20 \log (\mathrm{V}+6)
$$

is used, where $\mathrm{dB}$ represents the amplitude in decibels, $V$ is the value in volts, and a correction factor of +6 is used to avoid taking the logarithm of a negative or frac- 


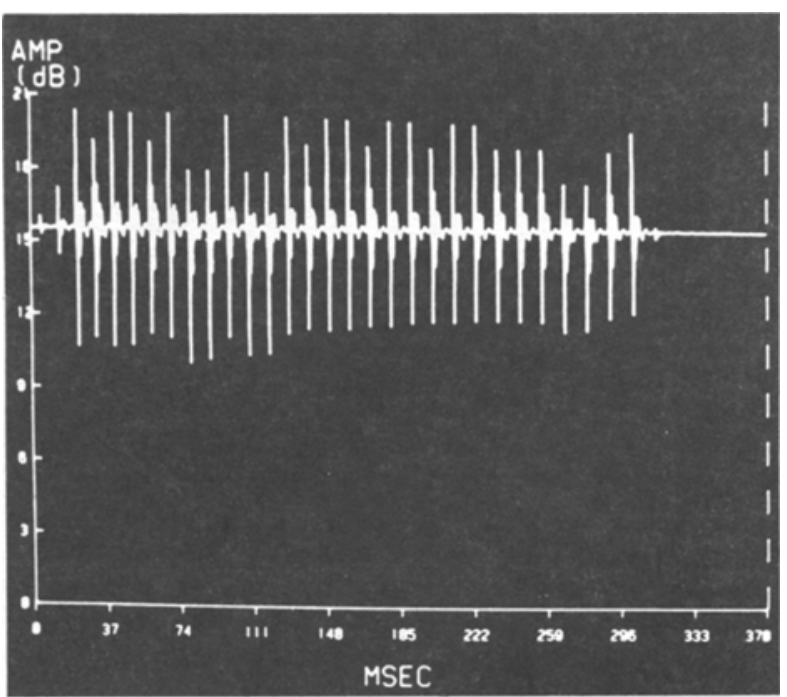

Figure 6. The full 370-msec synthetic vowel /a/ displayed with a decibel scale on the $y$-axis.

tional number. The resulting scale ranges from just under $20 \mathrm{~dB}$ to $0 \mathrm{~dB}$ (i.e., $20 \log 11$ to $20 \log 1$ ).

To convert the amplitude in either voltage or decibels to a height above the $x$-axis, the percentage

\section{(AMP - LOWAMP)/RANGE}

is computed, where (AMP - LOWAMP) is the height of the current amplitude and RANGE is the total amplitude range. This percentage is then multiplied by the total number of $y$-axis pixels (630) to obtain the height above the $\mathrm{x}$-axis.

\section{Listen to Waveform Segment}

When this option is selected, the currently displayed waveform is passed to an assembly language subroutine (PLYBCK). PLYBCK is a software handler for the DAC, and the DAC is connected to a laboratory loudspeaker via a low-pass filter (cutoff at $4.0 \mathrm{kHz}$, Butterworth characteristic, rolloff $48 \mathrm{~dB} /$ octave) and power amplifier. It should be noted that for time-shared operating systems, such as RSX-11M, the activity of other users can interrupt the audio-playback process unless execution priorities are changed. A further discussion of this and other system-specific issues is deferred to the next section.

\section{Discrete Fourier Transform}

In speech analysis, it is often desirable to view the speech signal in the frequency domain. A plethora of software exists for this purpose, and we selected an assembly language routine (Day, 1974) available through the Digital Equipment User's Society (DECUS). This routine performs a radix-2 fast Fourier transform (FFT) on $N$ data points, where $N$ is a power of 2 in the range $16 \leq N \leq 1,024$ (a 2,048-point option is available but is not used in this editor).

The data must be supplied to the FFT in 16-bit integer form, with a decimal point assumed between the two most significant bits, and Bit 16 is a sign bit. Thus, input values are normalized to the interval -1 to +1 , and are mapped onto this interval simply by dividing the voltages by 5 .

When invoked, the FFT routine computes the power spectrum and returns the spectral data in the upper half of the array that supplied the data to the FFT. The spectral data that emerge from the FFT are real in the interval 0 to 1 . The spectral data represent power amplitudes corresponding to frequencies of $i$ cycles per $N$ data points, where $i$ ranges from 0 to $N / 2$. The power spectrum is plotted from the spectral data with frequency $(0-5 \mathrm{kHz})$ on the $\mathrm{x}$-axis and decibels on the $\mathrm{y}$-axis.

The editor software provides two different ways of plotting the power spectrum. The first of these treats the FFT segments as discrete entities, plotting the power spectrum for each $N$-point segment. The current speech segment that is input to the FFT is broken into $N$-point subsegments with overlap. Hence, the first subsegment corresponds to the first $N$ points of the segment, the second subsegment runs from $N / 2$ to $3 N / 2$ points from the beginning, and so forth. The last subsegment, if it overshoots the end of the segment, is padded with values corresponding to $0 \mathrm{~V}$ (e.g., 2,047) until the end of the $N$ points required is reached. After the first of these subsegments is passed to the FFT, the function $P$ (freq) (a log relative power scale with values ranging from 0 to $\infty)$ is plotted in the range $0 \mathrm{~dB}$ to $-60 \mathrm{~dB}$ on the $y$-axis. $P$ is defined as

$$
P[10 *(i / N) \mathrm{kHz}]=10 \log A(i), i=0,1, \ldots, N / 2,
$$

where $A(i)$ is the power spectrum estimate described above. The $A(i)$ values are plotted in Figure 7 for a 512point FFT on the waveform shown in Figure 2. This plot is obviously only for the first 512 points of Figure 2, but the user can continue to iteratively plot successive transforms or can elect to return to the main menu at any time.

The second way of representing the spectral estimates attempts to emulate the speech spectrogram, by comput-

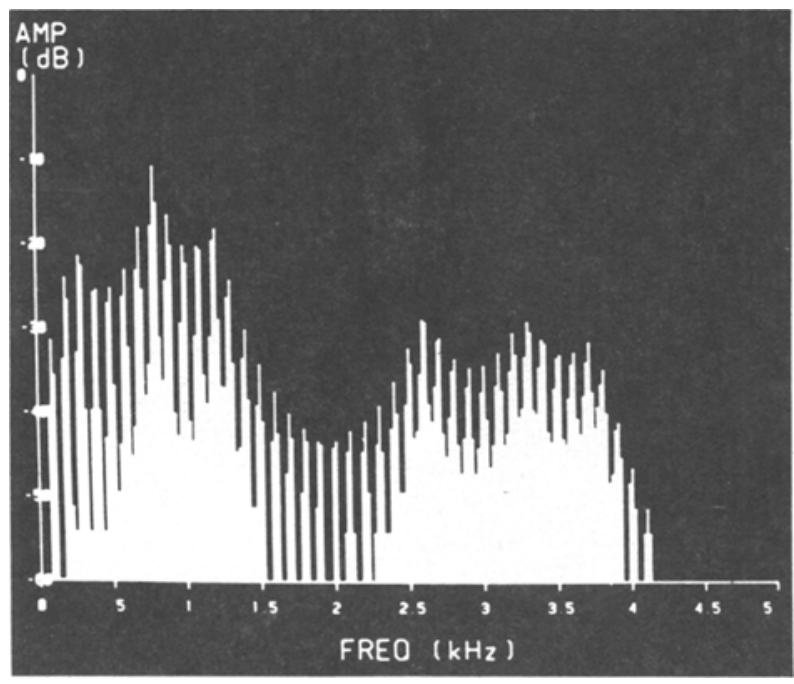

Figure 7. Display of the first 512-point power spectrum estimates for the synthetic vowel /a/. 
ing the FFT for all of the $N$-point segments and displaying the results with frequency on the $y$-axis and time (in milliseconds) on the $\mathrm{x}$-axis. The results of each transform are converted into horizontal line segments whose relative lengths are dependent on the magnitude of $P$ (freq) obtained from a particular transform. The line segments are centered in the first half of the transform segment, so that overlapping transforms do not yield overlapping visual information. The results of this operation are shown in Figure 8 for the entire speech segment shown in Figure 2. Figure 9 shows a transform done on sinusoidal data, and more clearly represents the intended effect.

The length of each horizontal line shown in Figures 8 and 9 is quantitatively computed as follows. First, all the transform segments are computed so that $\max [P($ freq) $)]$ is obtained. $\operatorname{Max}[P$ (freq) $]$ is then normalized to span the entire first-half range for its transform, and all others are scaled according to

PCTRNG $=[P$ (current $)-(-60)] /[P(\max )-(-60)]$.

The constant -60 is subtracted, since -60 is the minimum plottable value for $P$ (freq). After the spectrogram has been plotted, the user is returned to the main menu for further editing and/or analysis.

Finally, the user can specify any one of several windows to be imposed on the signal before performing the FFT. The window type for all transforms is user-selected when the FFT routine is entered. The windows currently available in the editor are: rectangular, triangular, Hanning, Hamming, Blackman, and Kaiser. The selection of a window significantly affects the results of the FFT, and the user should review the relevant signal-processing literature if unfamiliar with these techniques.

\section{SYSTEM REQUIREMENTS}

The software described above is designed to meet the general-purpose requirements for psychologically oriented

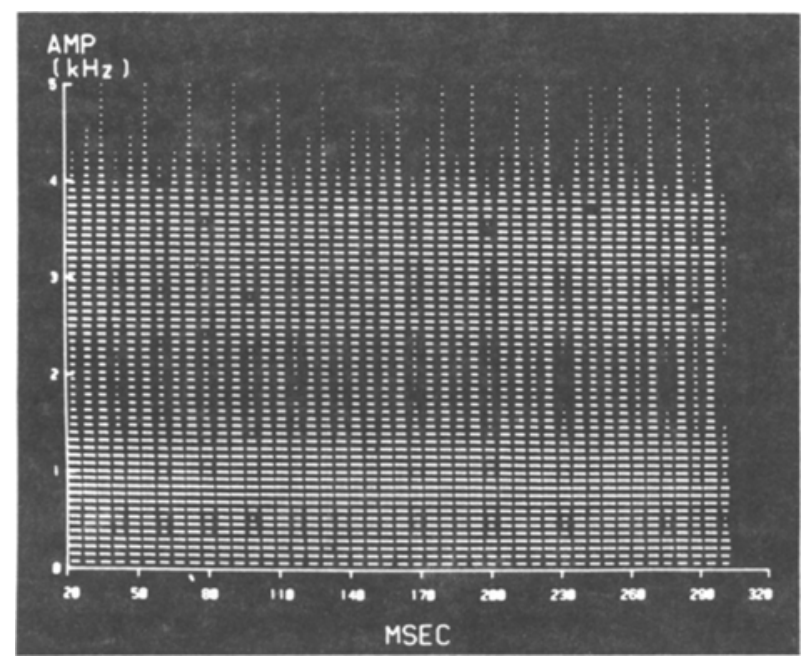

Figure 8. Emulation of a speech spectrogram for the 370-msec synthetic $/ \mathbf{a} /$.

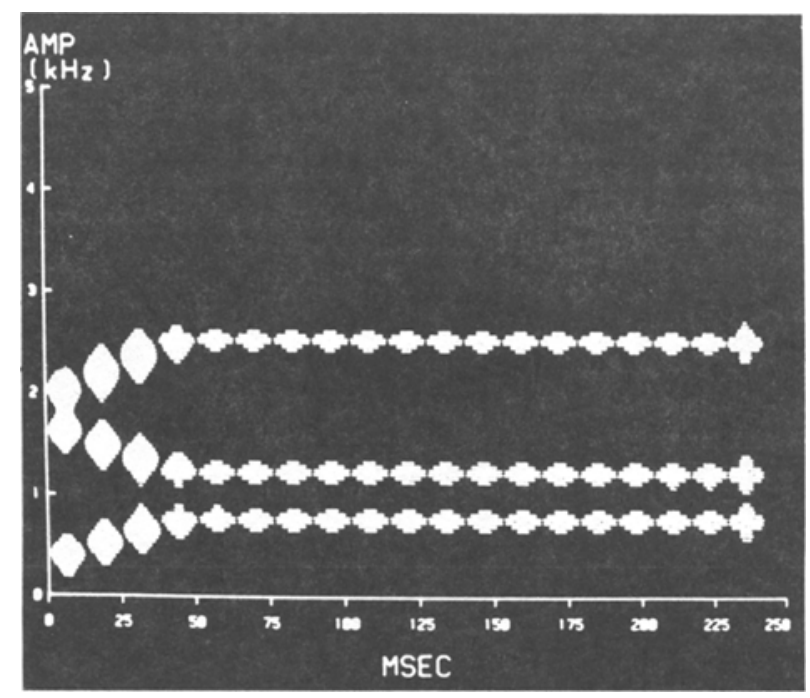

Figure 9. Emulation of a speech spectrogram for a quasispeech segment representing the consonant-vowel pair/ga/ with sinusoidal formant tracks.

speech research. However, it is necessary to briefly describe the system-dependent aspects of the software so that intersystem portability will be expedited.

\section{The RSX-11M Operating System}

Due to the relatively small amount of user space generally allowable under RSX-11M (32 kwords), the waveform editor had to be written as a main module that calls disk-resident subroutines to perform the basic operations. Therefore, the overlay capability of RSX-11M was used. As a normal task under RSX, the program WFORM.FTN and its subroutines would greatly exceed the $32 \mathrm{kword}$ limit imposed by RSX. In our scheme, the main module, common blocks, and a few selected subroutines reside in main memory at all times. The remainder of the available memory space is used to contain only the subroutine currently being called. Therefore, the size of available memory space will depend on the size of the largest subroutine. The overlay description (given in DEC's Overlay Description Language) is shown in Table 1.

The subroutines that drive the DAC, the clock (a KWV$11 \mathrm{C}$ clock board manufactured by DEC), and the DRV$11 \mathrm{~J}$ parallel I/O board were written as handlers and not installed in the operating system as device drivers. Therefore, for implementation on a similar DEC system and operating system, a memory partition must be created in high memory on the I/O page. The exact addresses for this partition depend on the particular system configuration.

The subroutine PLYBCK, when used with multiuser implementations of RSX (or other operating systems), must be raised to maximum available priority during execution. Otherwise, the activity of other users (or the operating system itself) will interrupt the handler and, thereby, interrupt the speech playback. On our system, we raised the priority of PLYBCK to 249, and disabled the line time 
Table 1

ODL (Overlay Description Language) Description of Waveform-Editing Software

\begin{tabular}{ll}
\hline & .ROOT CNTRL - *(A,B,C,D,E,F,G,H,I) \\
CNTRL: & .FCTR WFORM-BUFAP-HPDRV \\
$\mathrm{A}:$ & .FCTR AXES \\
$\mathrm{B}:$ & .FCTR FFTN-*(FFT, WINDOZ) \\
$\mathrm{I}:$ & .FCTR XAXIS \\
$\mathrm{C}:$ & .FCTR WPLOT-UTIL \\
$\mathrm{D}:$ & .FCTR FILEIN \\
$\mathrm{E}:$ & .FCTR SCRFIL \\
$\mathrm{F}:$ & .FCTR RESOLV \\
$\mathrm{G}:$ & .FCTR FBUILD \\
$\mathrm{H}:$ & .FCTR PLYBCK \\
& .END \\
\hline
\end{tabular}

clock (the clock that interrupts the processor every $1 / 60 \mathrm{sec}$ to provide round-robin user task scheduling).

\section{The H-P 1351A and 1311B Graphics System}

By far the most restrictive requirement for the editing system is the dependence on the H-P graphics system. The following description should permit the adaptation of the software for other graphic display systems.

The instruction set used by the 1351A controller is the H-P Graphics Generator Machine Language (GMML), a device-dependent language that consists of 16-bit octal values (some of which have arguments ANDed into the least significant bits of the instruction). Descriptions of this language are availble in the $1351 \mathrm{~A}$ Graphics Generator Operating and Service Manual (Hewlett-Packard, 1981) and in WFORM source code. Input from the LSI$11 / 23$ to the $1351 \mathrm{~A}$ is performed via the DRV-11J parallel $\mathrm{I} / \mathrm{O}$ board. It was convenient to buffer a sequence of instructions and data (common block /HPBUF/), and deliver the entire buffer at once by flushing /HPBUF/ down to the controller. The subroutine BUFAP performs the buffering and flush operations, and output is controlled by the subroutine HPDRV. HPDRV merely sends instructions or data one word at a time down the communications link and monitors the handshaking between the two devices.

The H-P instruction set controls the behavior of the vector graphics display. Among other things, the instructions control whether the "pen" is up or down and control the file structure within the controller memory. Actually, the controller file structure is the most distinctive feature of the 1351A. The 1351A has 8 kwords of on-board memory in which data and instructions can be stored. The programmer can partition the on-board memory into several files, and manipulate these files as separate entities. This permits selective erasure of portions of the display, separate handling of alphanumeric characters for labeling, and dynamic updating of the screen contents.

\section{AVAILABILITY}

Copies of the source code are available from the first author at no charge. Copies on magnetic tape will be provided on receipt of a replacement tape.

\section{REFERENCES}

BoGgs, G. J., AlLEY, R., \& GUZDIAL, M. (1984). Digital speech recording system using a multiprocessor architecture. Behavior Research Methods, Instruments, \& Computers, 16, 420-424.

DAY, R. (1974). Fast Fourier transform routine. Digital Equipment Company User's Society Program Library (No. 11-179). Maynard, MA: DECUS.

HEWLETT-PACKARD. (1981). 1351 A Graphics generator operating and service manual. Colorado Springs, CO: Author.

KLATT, D. H. (1979). Software for a cascade/parallel formant synthesizer. Journal of the Acoustical Society of America, 67, 914-953. 